\title{
ANTI-DIFFUSIVE HIGH ORDER WENO SCHEMES FOR HAMILTON-JACOBI EQUATIONS*
}

\author{
ZHENGFU XU $\mathrm{XU}^{\dagger}$ AND CHI-WANG SHU ${ }^{\ddagger}$
}

\section{Dedicated to Professor Joel A. Smoller on the occasion of his 70th birthday}

\begin{abstract}
In this paper, we generalize the technique of anti-diffusive flux corrections for high order finite difference WENO schemes solving conservation laws in [21], to solve Hamilton-Jacobi equations. The objective is to obtain sharp resolution for kinks, which are derivative discontinuities in the viscosity solutions of Hamilton-Jacobi equations. We would like to resolve kinks better while maintaining high order accuracy in smooth regions. Numerical examples for one and two space dimensional problems demonstrate the good quality of these Hamiltonian corrected WENO schemes.
\end{abstract}

Key words. Hamilton-Jacobi equations, anti-diffusive flux correction, kinks, Hamiltonian correction, high order accuracy, finite difference, WENO scheme

AMS subject classifications. $65 \mathrm{M} 06$

1. Introduction. In this paper, we consider the numerical solutions of the Hamilton-Jacobi equations

$$
u_{t}+H\left(u_{x_{1}}, \cdots, u_{x_{n}}\right)=0, \quad u(x, 0)=u_{0}(x) .
$$

As is well known, solutions to (1.1) are Lipschitz continuous but could contain discontinuous derivatives, even when the initial conditions are smooth. Derivative discontinuities are observed as kinks in geometric structures. The nonuniqueness of solutions to (1.1) also makes it necessary to define the concept of a viscosity solution, to single out a unique, practically relevant solution. See Crandall and Lions [4].

In [5], Crandall and Lions proved convergence for first order monotone finite difference schemes to the viscosity solutions of (1.1). To solve (1.1) with higher order accuracy, Osher and Sethian in [15] and Osher and Shu in [16] introduced a class of high order essentially non-oscillatory (ENO) schemes, which are adapted from the methodologies for hyperbolic conservation laws [7, 19, 20]. Weighted ENO (WENO) schemes for conservation laws $[14,10]$ are adapted to solve the Hamilton-Jacobi equations in [9] for the rectangular meshes and in [22] for the triangular meshes. RungeKutta discontinuous Galerkin methods for conservation laws [3, 2] are also adapted to solve the Hamilton-Jacobi equations in $[8,11,13]$. Numerical results produced by these high order schemes indicate convergence to the viscosity solutions of (1.1) with high order accuracy in smooth regions and sharp resolutions of the derivative discontinuities.

We now concentrate on the issue of sharp resolutions of the derivative discontinuities, which is the main theme of this paper. High order finite difference WENO schemes for solving Hamilton-Jacobi equations [9] do produce better resolutions for

*Received May 3, 2005; accepted for publication November 30, 2005. Research supported by ARO grant W911NF-04-1-0291, NSF grants DMS-0207451 and DMS-0510345, and AFOSR grant FA9550-05-1-0123.

${ }^{\dagger}$ Department of Mathematics, Pennsylvania State University, University Park, PA 16802, USA (xu_z@math.psu.edu).

${ }^{\ddagger}$ Division of Applied Mathematics, Brown University, Providence, Rhode Island 02912, USA (shu@dam.brown.edu). 
the derivative discontinuities than a low order scheme, especially for nonlinear problems. However, for linear problems, numerical results by high order schemes still suffer from relatively poor resolutions of discontinuous derivatives comparing with the resolution for nonlinear problems. This is a more serious issue for long time evolution, when the resolution of kinks becomes progressively worse. Considering the close link between Hamilton-Jacobi equations and conservation laws, it is natural to adapt techniques of sharpening contact discontinuities for conservation laws to obtain sharper resolution of derivative discontinuities for Hamilton-Jacobi equations. In this paper, we extend our work in [21] of anti-diffusive flux corrections for high order finite difference WENO schemes for conservation laws to solve Hamilton-Jacobi equations. The technique we explored in [21] for sharpening contact discontinuities is adapted to resolve derivative discontinuities for linear Hamilton-Jacobi equations both in one space dimension and in two space dimensions.

This paper is organized as follows. In Section 2, we review briefly the antidiffusive flux correction technique developed in [21], in a finite volume framework rather than in a finite difference framework as in [21]. This technique in a finite volume framework for solving conservation law equations is then adapted to solve one dimensional Hamilton-Jacobi equations in Section 3. In Section 4, we generalize this Hamiltonian correction method to two (or higher) dimensional Hamilton-Jacobi equations. We demonstrate the good numerical quality achieved by this Hamiltonian correction method through extensive numerical examples in Section 5. Concluding remarks are given in Section 6.

2. Flux corrections for high order finite volume WENO schemes. In this section, we briefly review the flux correction technique developed and applied in [21] for solving conservation law equations both in one dimension and in two dimensions. However, instead of describing the technique in a finite difference framework as in [21], we present the technique in a finite volume framework which is easier to adapt to solve the Hamilton-Jacobi equations in next section.

2.1. Flux corrections for finite volume WENO schemes in one dimension. We consider the one dimensional conservation law

$$
u_{t}+f(u)_{x}=0
$$

with $f^{\prime}(u)>0$, for simplicity. The case with $f^{\prime}(u)<0$ can be considered symmetrically. We reintroduce the flux correction technique in [21] by a third order TVD Runge-Kutta method in time and a fifth order finite volume WENO discretization in space, instead of the finite difference WENO discretization adopted in [21]. Notice that the choice of fifth order WENO discretization in space and third order RungeKutta method in time is just for the convenience of presentation: the flux correction technique can be applied to WENO schemes of any order and also to other RungeKutta methods. The full algorithm has the following form

$$
\begin{aligned}
\bar{u}^{(1)} & =\bar{u}^{n}+\Delta t L\left(\bar{u}^{n}\right) \\
\bar{u}^{(2)} & =\bar{u}^{n}+\frac{1}{4} \Delta t L\left(\bar{u}^{n}\right)+\frac{1}{4} \Delta t L\left(\bar{u}^{(1)}\right) \\
\bar{u}^{n+1} & =\bar{u}^{n}+\frac{1}{6} \Delta t L\left(\bar{u}^{n}\right)+\frac{1}{6} \Delta t L\left(\bar{u}^{(1)}\right)+\frac{2}{3} \Delta t L\left(\bar{u}^{(2)}\right)
\end{aligned}
$$

where $\bar{u}$ denotes the cell average of the solution $u, \Delta t$ is the time step which can be changed from step to step, and $L$ denotes the spatial WENO operator to be described 
in detail below. In order to maintain the moving traveling wave solutions for piecewise constant functions containing contact discontinuities, we modified the scheme (2.2) in [21] by

$$
\begin{aligned}
\bar{u}^{(1)} & =\bar{u}^{n}+\Delta t L\left(\bar{u}^{n}\right) \\
\bar{u}^{(2)} & =\bar{u}^{n}+\frac{1}{4} \Delta t L^{\prime}\left(\bar{u}^{n}\right)+\frac{1}{4} \Delta t L\left(\bar{u}^{(1)}\right) \\
\bar{u}^{n+1} & =\bar{u}^{n}+\frac{1}{6} \Delta t L^{\prime \prime}\left(\bar{u}^{n}\right)+\frac{1}{6} \Delta t L\left(\bar{u}^{(1)}\right)+\frac{2}{3} \Delta t L\left(\bar{u}^{(2)}\right)
\end{aligned}
$$

where the operator $L$ is defined by

$$
L(\bar{u})_{i}=-\lambda_{i}\left(\hat{f}_{i+\frac{1}{2}}^{a}-\hat{f}_{i-\frac{1}{2}}^{a}\right),
$$

with $\lambda_{i}=\frac{\Delta t}{\Delta x_{i}}$, where $\Delta x_{i}$ is the local mesh size of cell $i$, and the anti-diffusive numerical flux $\hat{f}^{a}$ is given by

$\hat{f}_{i+\frac{1}{2}}^{a}=f\left(u_{i+\frac{1}{2}}^{-}\right)+\varphi_{i} \operatorname{minmod}\left(\frac{\bar{u}_{i}-\bar{u}_{i-1}}{\lambda_{i}}+f\left(u_{i-\frac{1}{2}}^{-}\right)-f\left(u_{i+\frac{1}{2}}^{-}\right), f\left(u_{i+\frac{1}{2}}^{+}\right)-f\left(u_{i+\frac{1}{2}}^{-}\right)\right)$

where $u_{i+\frac{1}{2}}^{ \pm}$are the high order WENO reconstructions of the point values of $u$ from its cell averages $\bar{u}$, with stencils biasing to the left and to the right respectively, see $[10,18]$ for details. The minmod function is defined as usual

$$
\operatorname{minmod}(a, b)=\left\{\begin{array}{lll}
0 & a b \leq 0 \\
a & a b>0, & |a| \leq|b| \\
b & a b>0, & |a|>|b|
\end{array}\right.
$$

The operator $L^{\prime}$ is defined by

$$
L^{\prime}(\bar{u})_{i}=-\lambda_{i}\left(\bar{f}_{i+\frac{1}{2}}^{a}-\bar{f}_{i-\frac{1}{2}}^{a}\right)
$$

with the modified anti-diffusive flux $\bar{f}^{a}$ given by

$\bar{f}_{i+\frac{1}{2}}^{a}=\left\{\begin{array}{l}f\left(u_{i+\frac{1}{2}}^{-}\right)+\varphi_{i} \operatorname{minmod}\left(\frac{4\left(\bar{u}_{i}-\bar{u}_{i-1}\right)}{\lambda_{i}}+f\left(u_{i-\frac{1}{2}}^{-}\right)-f\left(u_{i+\frac{1}{2}}^{-}\right), f\left(u_{i+\frac{1}{2}}^{+}\right)-f\left(u_{i+\frac{1}{2}}^{-}\right)\right) \\ \hat{f}^{a}\end{array}\right.$

corresponding to the cases

$$
\left\{\begin{array}{l}
b c>0,|b|<|c| \\
\text { otherwise }
\end{array}\right.
$$

respectively, and the operator $L^{\prime \prime}$ is defined by

$$
L^{\prime \prime}(\bar{u})_{i}=-\lambda_{i}\left(\tilde{f}_{i+\frac{1}{2}}^{a}-\tilde{f}_{i-\frac{1}{2}}^{a}\right)
$$

with the modified anti-diffusive flux $\tilde{f}^{a}$ given by

$$
\tilde{f}_{i+\frac{1}{2}}^{a}=\left\{\begin{array}{l}
f\left(u_{i+\frac{1}{2}}^{-}\right)+\varphi_{i} \operatorname{minmod}\left(\frac{6\left(\bar{u}_{i}-\bar{u}_{i-1}\right)}{\lambda_{i}}+f\left(u_{i-\frac{1}{2}}^{-}\right)-f\left(u_{i+\frac{1}{2}}^{-}\right), f\left(u_{i+\frac{1}{2}}^{+}\right)-f\left(u_{i+\frac{1}{2}}^{-}\right)\right) \\
\hat{f}^{a}
\end{array}\right.
$$


corresponding to the cases (2.6) respectively. Here $b$ and $c$ are defined as $b=\frac{\bar{u}_{i}-\bar{u}_{i-1}}{\lambda_{i}}+$ $f\left(u_{i-\frac{1}{2}}^{-}\right)-f\left(u_{i+\frac{1}{2}}^{-}\right), c=f\left(u_{i+\frac{1}{2}}^{+}\right)-f\left(u_{i+\frac{1}{2}}^{-}\right)$and $\varphi_{i}$ is a discontinuity indicator with its value between 0 and 1 . Ideally, $\varphi_{i}$ should be close to 0 in smooth regions and close to 1 near a discontinuity. Our original choice of $\varphi_{i}$ can be found in [21] and a somewhat improved choice is described in Section 2.2. We remark that the scheme (2.3) with $L, L^{\prime}$ and $L^{\prime \prime}$ defined by $(2.4),(2.5)$ and (2.7) is fifth order accurate in space and third order accurate in time. The correction to the original WENO scheme is no larger in magnitude than that of $f\left(u_{i+\frac{1}{2}}^{+}\right)-f\left(u_{i+\frac{1}{2}}^{-}\right)$, which is on the level of truncation errors for the WENO schemes because both $u_{i+\frac{1}{2}}^{+}$and $u_{i+\frac{1}{2}}^{-}$are high order approximations to the same point value $u\left(x_{i+\frac{1}{2}}\right)$. This ensures that the high order accuracy of the finite volume WENO schemes is achieved. The purpose of the extra factor 4 in the first argument of the minmod function in the definition of $\bar{f}^{a}$ and the extra factor 6 in the first argument of the minmod function in the definition of $\tilde{f}^{a}$ is to compensate for the coefficients $\frac{1}{4}$ and $\frac{1}{6}$ in front of $L^{\prime}$ and $L^{\prime \prime}$ respectively, so that the final scheme could still maintain exactly traveling wave solutions of a piecewise constant function. We refer to [21] for more details.

For the upwind WENO-Roe schemes [10], the numerical flux is chosen as $f\left(u_{i+\frac{1}{2}}^{-}\right)$ when $f^{\prime}(u)>0$ and as $f\left(u_{i+\frac{1}{2}}^{+}\right)$when $f^{\prime}(u) \leq 0$. The scheme described above is therefore a flux corrected WENO-Roe scheme. Due to the entropy violating possibility of the Roe scheme, we only apply this technique on linear problems or linearly degenerate fields of systems.

2.2. The discontinuity indicator. The discontinuity indicator $\varphi_{i}$ is designed such that it is close to 0 in smooth regions and close to 1 near a discontinuity. Out of symmetry consideration and for the objective of a better description of the discontinuity positions, we modify the definition of $\varphi_{i}$ in [21] slightly to the following form:

$$
\varphi_{i}=\frac{\beta_{i}}{\beta_{i}+\gamma_{i}}
$$

with

$$
\begin{gathered}
\alpha_{i}=\left|\bar{u}_{i-1}-\bar{u}_{i}\right|^{2}+\varepsilon, \quad \xi_{i}=\left|\bar{u}_{i-1}-\bar{u}_{i+1}\right|^{2}+\varepsilon \\
\beta_{i}=\frac{\xi_{i}}{\alpha_{i-1}}+\frac{\xi_{i}}{\alpha_{i+2}}, \quad \gamma_{i}=\frac{\left|\bar{u}_{\max }-\bar{u}_{\min }\right|^{2}}{\alpha_{i}} .
\end{gathered}
$$

Here $\varepsilon$ is a small positive number taken as $10^{-6}$ in our numerical experiments, and $\bar{u}_{\max }$ and $\bar{u}_{\min }$ are the maximum and minimum values of $\bar{u}_{j}$ for all cells. Clearly, $0 \leq \varphi_{i} \leq 1$, and $\varphi_{i}=O\left(\Delta x^{2}\right)$ in smooth regions. Near a strong discontinuity, $\gamma_{i} \ll \beta_{i}, \varphi_{i}$ is close to 1 .

2.3. Flux corrections for finite volume WENO schemes in two dimensions. Two dimensional finite volume WENO schemes are similar to the schemes for one dimension described above, with reconstruction from two dimensional cell averages to point values, which can be performed sequentially one dimension after another for rectangular meshes. We refer to $[17,18]$ for more details. Thus, for example, after the reconstruction of the point values $u_{i+\frac{1}{2}, j}$ and $u_{i, j+\frac{1}{2}}$ at the interfaces, for the equation

$$
u_{t}+f(u)_{x}+g(u)_{y}=0, \quad f^{\prime}(u)>0, \quad g^{\prime}(u)>0,
$$


we present the anti-diffusive flux by

$$
\begin{aligned}
\hat{f}_{i+\frac{1}{2}, j}^{a} & =f\left(u_{i+\frac{1}{2}, j}^{-}\right) \\
& +\varphi_{i, j} \operatorname{minmod}\left(\frac{\bar{u}_{i, j}-\bar{u}_{i-1, j}}{d \lambda_{i}^{x}}+f\left(u_{i-\frac{1}{2}, j}^{-}\right)-f\left(u_{i+\frac{1}{2}, j}^{-}\right), f\left(u_{i+\frac{1}{2}, j}^{+}\right)-f\left(u_{i+\frac{1}{2}, j}^{-}\right)\right)
\end{aligned}
$$

where $d=2$ is the dimension. For fixed $j, \varphi_{i, j}$ has the same definition as in (2.8) in one dimension. Symmetrically for the $y$ direction, we have

$$
\begin{aligned}
\hat{g}_{i, j+\frac{1}{2}}^{a} & =g\left(u_{i, j+\frac{1}{2}}^{-}\right) \\
& +\psi_{i, j} \operatorname{minmod}\left(\frac{\bar{u}_{i, j}-\bar{u}_{i, j-1}}{d \lambda_{j}^{y}}+g\left(u_{i, j-\frac{1}{2}}^{-}\right)-g\left(u_{i, j+\frac{1}{2}}^{-}\right), g\left(u_{i, j+\frac{1}{2}}^{+}\right)-g\left(u_{i, j+\frac{1}{2}}^{-}\right)\right)
\end{aligned}
$$

with the discontinuity indicator $\psi_{i, j}$ defined similarly to the one dimensional case in (2.8) in the $y$ direction with fixed $x_{i}$. The procedure described above applies to each quadrature point along the cell interfaces to compute the numerical fluxes.

3. Hamiltonian corrected method for one dimensional Hamilton-Jacobi equations. In this section, we consider the one dimensional Hamilton-Jacobi equation

$$
u_{t}+H\left(u_{x}\right)=0
$$

with $H^{\prime}(u)>0$, for simplicity. The other case $H^{\prime}(u)<0$ can be considered symmetrically. The tight link between the Hamilton-Jacobi equation and the conservation law equation (2.1) in one dimension makes it easy to adapt schemes for conservation laws to schemes for Hamilton-Jacobi equations [15]. We will adapt the flux correction technique, developed in [21] and reviewed in the previous section, for sharpening contact discontinuities of high order WENO schemes for solving conservation laws, to sharpen kinks in the solutions of linear Hamilton-Jacobi equations. We start with the adaptation of the first order scheme in [6] to the Hamilton-Jacobi equation.

3.1. First order Hamiltonian corrected method. We use the explicit form, interpreted in [1], of the limited down-wind scheme reintroduced in [6]. We refer the details of the limited down-wind scheme to [6]. Its simple explicit equivalent finite volume scheme for the conservation law (2.1) with $f^{\prime}(u)>0$ has the following definition for the numerical flux

$$
\hat{f}_{i+\frac{1}{2}}^{a}=f\left(\bar{u}_{i}\right)+\operatorname{minmod}\left(\frac{\bar{u}_{i}-\bar{u}_{i-1}}{\lambda_{i}}+f\left(\bar{u}_{i-1}\right)-f\left(\bar{u}_{i}\right), f\left(\bar{u}_{i+1}\right)-f\left(\bar{u}_{i}\right)\right) .
$$

A relaxed version of $(3.2)$ we have used is

$$
\hat{f}_{i+\frac{1}{2}}^{a}=f\left(\bar{u}_{i}\right)+\varphi_{i} \operatorname{minmod}\left(\frac{\bar{u}_{i}-\bar{u}_{i-1}}{\lambda_{i}}+f\left(\bar{u}_{i-1}\right)-f\left(\bar{u}_{i}\right), f\left(\bar{u}_{i+1}\right)-f\left(\bar{u}_{i}\right)\right),
$$

where $\varphi_{i}$ is the discontinuity indicator given by (2.8).

This first order anti-diffusive scheme has the following property, which was proved in $[6,1]$.

Proposition 3.1. Assume that the CFL condition $\lambda_{i} f^{\prime}(u) \leq 1$ holds, and the numerical flux is defined by (3.3), then the scheme

$$
\bar{u}_{i}^{n+1}-\bar{u}_{i}^{n}+\lambda_{i}\left(\hat{f}_{i+\frac{1}{2}}^{n}-\hat{f}_{i-\frac{1}{2}}^{n}\right)=0
$$


satisfies the maximum principle

$$
\min _{k} \bar{u}_{k}^{n} \leq \bar{u}_{i}^{n+1} \leq \max _{k} \bar{u}_{k}^{n} .
$$

To solve the Hamilton-Jacobi equation (3.1), we define the first order divided difference operator $D_{-}$by

$$
D_{-} u_{i}=\frac{u_{i}-u_{i-1}}{\Delta x}
$$

where $u_{i}=u\left(x_{i}\right)$ is the point value of the solution $u$ and for simplicity we assume that the mesh size $\Delta x$ is a constant. This operator actually defines a cell average for $u_{x}$ as it equals $\frac{1}{\Delta x} \int_{x_{i-1}}^{x_{i}} u_{x} d x$. We adapt the finite volume scheme (3.4) and obtain the following corrected numerical Hamiltonian

$$
\begin{aligned}
\hat{H}_{i}^{a} & =H\left(D_{-} u_{i}\right) \\
& +\varphi_{i} \operatorname{minmod}\left(\frac{D_{-} u_{i}-D_{-} u_{i-1}}{\lambda_{i}}+H\left(D_{-} u_{i-1}\right)-H\left(D_{-} u_{i}\right), H\left(D_{-} u_{i+1}\right)-H\left(D_{-} u_{i}\right)\right)
\end{aligned}
$$

to form the following scheme

$$
u_{i}^{n+1}-u_{i}^{n}+\Delta t \hat{H}_{i}^{a}=0
$$

for solving the Hamilton-Jacobi equation (3.1). Parallel to Proposition 3.1, we have the following property for this first order Hamiltonian corrected scheme:

Proposition 3.2. Assume that the CFL condition $\lambda_{i} H^{\prime}(u) \leq 1$ holds, and the numerical Hamiltonian is defined as in (3.5), then the scheme (3.6) satisfies the maximum principle for the first order divided difference

$$
\min _{k} D_{-} u_{k}^{n} \leq D_{-} u_{i}^{n+1} \leq \max _{k} D_{-} u_{k}^{n} .
$$

For the proof, we simply need to take the operator $D_{-}$on (3.6) and then use Proposition 3.1.

An immediate conclusion we can reach from Proposition 3.2 is

Proposition 3.3. Assume that the CFL condition $\lambda_{i} H^{\prime}(u) \leq 1$ holds, and the numerical Hamiltonian is defined as in (3.5), then the scheme (3.6) is total variation bounded (TVB)

$$
\sum_{i=1}^{N}\left|u_{i}^{n}-u_{i-1}^{n}\right| \leq L \max _{i}\left|D_{-} u_{i}^{0}\right|
$$

for any time level $n$, where $L$ is the length of the domain for computation. 
3.2. High order Hamiltonian corrected method. Using the first order divided differences $D_{-} u_{i}$ and using the standard WENO procedure, we can reconstruct $D_{-}^{-} u_{i}$ and $D_{-}^{+} u_{i}$ as the left and right high order approximations of $u_{x}\left(x_{i}\right)$. This WENO reconstruction is given in details in [9]. We then adapt our anti-diffusive fifth order finite volume WENO scheme, presented in Section 2, to the following Hamiltonian corrected method for solving the Hamilton-Jacobi equation (3.1):

$$
\begin{aligned}
u^{(1)} & =u^{n}+\Delta t L\left(u^{n}\right) \\
u^{(2)} & =u^{n}+\frac{1}{4} \Delta t L^{\prime}\left(u^{n}\right)+\frac{1}{4} \Delta t L\left(u^{(1)}\right) \\
u^{n+1} & =u^{n}+\frac{1}{6} \Delta t L^{\prime \prime}\left(u^{n}\right)+\frac{1}{6} \Delta t L\left(u^{(1)}\right)+\frac{2}{3} \Delta t L\left(u^{(2)}\right)
\end{aligned}
$$

where the operator $L$ is defined by

$$
L(u)_{i}=-\hat{H}_{i}^{a}
$$

with the numerical Hamiltonian $\hat{H}^{a}$ given by

$$
\begin{aligned}
\hat{H}_{i}^{a} & =H\left(D_{-}^{-} u_{i}\right) \\
& +\varphi_{i} \operatorname{minmod}\left(\frac{D_{-} u_{i}-D_{-} u_{i-1}}{\lambda_{i}}+H\left(D_{-}^{-} u_{i-1}\right)-H\left(D_{-}^{-} u_{i}\right), H\left(D_{-}^{+} u_{i}\right)-H\left(D_{-}^{-} u_{i}\right)\right)
\end{aligned}
$$

The operator $L^{\prime}$ is defined by

$$
L^{\prime}(u)_{i}=-\bar{H}_{i}^{a}
$$

with the numerical Hamiltonian $\bar{H}^{a}$ given by

$\bar{H}_{i}^{a}=\left\{\begin{array}{l}H\left(D_{-}^{-} u_{i}\right)+\varphi_{i} \operatorname{minmod}\left(\frac{4\left(D_{-} u_{i}-D_{-} u_{i-1}\right)}{\lambda_{i}}+H\left(D_{-}^{-} u_{i-1}\right)-H\left(D_{-}^{-} u_{i}\right), H\left(D_{-}^{+} u_{i}\right)-H\left(D_{-}^{-} u_{i}\right)\right) \\ \hat{H}^{a}\end{array}\right.$

corresponding to the cases (2.6) respectively; the operator $L^{\prime \prime}$ is defined by

$$
L^{\prime \prime}(u)_{i}=-\tilde{H}_{i}^{a}
$$

with the numerical Hamiltonian $\tilde{H}^{a}$ given by

$\tilde{H}_{i}^{a}=\left\{\begin{array}{l}H\left(D_{-}^{-} u_{i}\right)+\varphi_{i} \operatorname{minmod}\left(\frac{6\left(D_{-} u_{i}-D_{-} u_{i-1}\right)}{\lambda_{i}}+H\left(D_{-}^{-} u_{i-1}\right)-H\left(D_{-}^{-} u_{i}\right), H\left(D_{-}^{+} u_{i}\right)-H\left(D_{-}^{-} u_{i}\right)\right) \\ \hat{H}^{a}\end{array}\right.$

corresponding to the cases (2.6) respectively, with $b=\frac{D_{-} u_{i}-D_{-} u_{i-1}}{\lambda_{i}}+H\left(D_{-}^{-} u_{i-1}\right)-$ $H\left(D_{-}^{-} u_{i}\right)$ and $c=H\left(D_{-}^{+} u_{i}\right)-H\left(D_{-}^{-} u_{i}\right)$. Finally, the kink indicator $\varphi_{i}$ is again given by (2.8), with the cell averages $\bar{u}$ in (2.9) replaced by the first order divided differences $D_{-} u$. This relaxation coefficient $\varphi_{i}$, which is a number between 0 and 1 , is again introduced to give correction to the numerical Hamiltonians around kinks, and meanwhile to inherit the original WENO scheme in smooth regions.

Once again, we remark that the scheme (3.9) is fifth order accurate in space (when the WENO reconstruction is fifth order accurate) and third order accurate in time. The correction to the original WENO Hamiltonian is no larger in magnitude than that of $H\left(D_{-}^{+} u_{i}\right)-H\left(D_{-}^{-} u_{i}\right)$, which is on the level of truncation errors for the WENO schemes because both $D_{-}^{+} u_{i}$ and $D_{-}^{-} u_{i}$ are high order approximations to the 
point value $u_{x}$ at the same location $x_{i}$. This guarantees that the high order accuracy of the WENO scheme is maintained. As before, the choice of fifth order WENO discretization in space and third order Runge-Kutta method in time is just for the convenience of presentation: the Hamiltonian correction technique can be applied to WENO schemes of any order and also to other Runge-Kutta methods.

4. Hamiltonian corrected method for two dimensional Hamilton-Jacobi equations. We have presented the flux corrected methods for high order finite difference and finite volume WENO schemes for solving two dimensional conservation laws in [21] and in Section 2, respectively. In this section, we directly adapt the anti-diffusive WENO schemes for two dimensional conservation law equations to two dimensional Hamilton-Jacobi equations

$$
u_{t}+H\left(x, y, u_{x}, u_{y}\right)=0 .
$$

For simplicity, we assume $\frac{\partial H}{\partial u_{x}}>0$ and $\frac{\partial H}{\partial u_{y}}>0$. Other cases can be treated symmetrically. Our Hamiltonian corrected scheme is given as follows

$$
\begin{aligned}
u^{(1)} & =u^{n}+\Delta t L\left(u^{n}\right) \\
u^{(2)} & =u^{n}+\frac{1}{4} \Delta t L^{\prime}\left(u^{n}\right)+\frac{1}{4} \Delta t L\left(u^{(1)}\right) \\
u^{n+1} & =u^{n}+\frac{1}{6} \Delta t L^{\prime \prime}\left(u^{n}\right)+\frac{1}{6} \Delta t L\left(u^{(1)}\right)+\frac{2}{3} \Delta t L\left(u^{(2)}\right)
\end{aligned}
$$

with the operator $L(u)$ defined by

$$
L(u)_{i j}=-\left(\hat{H}_{i, j}+C_{i, j}^{x}+C_{i, j}^{y}\right)
$$

where $\hat{H}$ is the upwind numerical Hamiltonian

$$
\hat{H}_{i, j}=H\left(x_{i}, y_{j}, D_{x}^{-} u_{i, j}, D_{y}^{-} u_{i, j}\right),
$$

and $C_{i, j}^{x}$ and $C_{i, j}^{y}$ are the Hamiltonian corrections in the $x$ and $y$ directions respectively, defined by

$$
\begin{aligned}
& C_{i, j}^{x}=\varphi_{i, j} \operatorname{minmod}\left(\frac{D_{x} u_{i, j}-D_{x} u_{i-1, j}}{d \cdot \lambda_{i}^{x}}+\hat{H}_{i-1, j}-\hat{H}_{i, j}, H\left(x_{i}, y_{j}, D_{x}^{+} u_{i, j}, D_{y}^{-} u_{i, j}\right)-\hat{H}_{i, j}\right) \\
& C_{i, j}^{y}=\psi_{i, j} \operatorname{minmod}\left(\frac{D_{y} u_{i, j}-D_{y} u_{i, j-1}}{d \cdot \lambda_{j}^{y}}+\hat{H}_{i, j-1}-\hat{H}_{i, j}, H\left(x_{i}, y_{j}, D_{x}^{-} u_{i, j}, D_{y}^{+} u_{i, j}\right)-\hat{H}_{i, j}\right)
\end{aligned}
$$

where $d=2$ is the dimension. Same as in the one dimensional case, we fix $j$ to define $D_{x} u_{i, j}$ as

$$
D_{x} u_{i, j}=\frac{u_{i, j}-u_{i-1, j}}{\Delta_{i}^{x}}
$$

and symmetrically, we fix $i$ to define $D_{y} u_{i, j}$ as

$$
D_{y} u_{i, j}=\frac{u_{i, j}-u_{i, j-1}}{\Delta_{j}^{y}} .
$$

We define the relaxation coefficients $\varphi_{i, j}$ and $\psi_{i, j}$ exactly the same way as in the one dimensional case in Section 3 by fixing $j$ and $i$ respectively. Finally, the modified 
TABLE 5.1

Errors and numerical orders of accuracy of the fifth order Hamiltonian corrected fifth order WENO scheme and the original fifth order WENO scheme for the one dimensional linear HamiltonJacobi equation (5.1).

\begin{tabular}{|c|c|c|c|c|c|c|c|c|}
\hline & \multicolumn{3}{|c|}{ Hamiltonian corrected WENO } & \multicolumn{4}{c|}{ original WENO } \\
\hline $\mathrm{N}$ & $L^{1}$ error & order & $L^{\infty}$ error & order & $L^{1}$ error & order & $L^{\infty}$ error & order \\
\hline 40 & $3.33 \mathrm{E}-6$ & & $5.34 \mathrm{E}-6$ & & $4.46 \mathrm{E}-5$ & & $7.72 \mathrm{E}-5$ & \\
\hline 80 & $1.39 \mathrm{E}-6$ & 4.58 & $2.20 \mathrm{E}-6$ & 4.60 & $1.48 \mathrm{E}-6$ & 4.91 & $2.41 \mathrm{E}-6$ & 5.00 \\
\hline 160 & $4.66 \mathrm{E}-8$ & 4.89 & $7.35 \mathrm{E}-8$ & 4.78 & $4.73 \mathrm{E}-8$ & 4.97 & $7.51 \mathrm{E}-8$ & 5.00 \\
\hline 320 & $1.48 \mathrm{E}-9$ & 4.97 & $2.34 \mathrm{E}-9$ & 4.97 & $1.49 \mathrm{E}-9$ & 4.99 & $2.35 \mathrm{E}-9$ & 4.99 \\
\hline 640 & $4.66 \mathrm{E}-11$ & 4.98 & $7.33 \mathrm{E}-11$ & 4.99 & $4.66 \mathrm{E}-11$ & 5.00 & $7.34 \mathrm{E}-11$ & 5.00 \\
\hline
\end{tabular}

operators $L^{\prime}$ and $L^{\prime \prime}$ can be defined similarly as before with the extra factors 4 and 6 in the first arguments of the minmod functions respectively under the condition (2.6).

Again, this is a high order scheme according to the same observation that the Hamiltonian correction is no larger in magnitude than the truncation error of the WENO reconstruction as in the one dimensional case. We will display numerical effect of this scheme in next section, especially its quality in capturing kinks.

5. Numerical results. We give extensive numerical examples in this section to demonstrate the effect of the schemes we have developed in previous sections. Notice that all PDEs in this section are understood to be the Hamilton-Jacobi equations and we seek approximations to the viscosity solutions, which are continuous but may contain discontinuous derivatives. The CFL number is taken as 0.3 , except for the accuracy tests where the CFL numbers are taken smaller for more refined meshes to guarantee that spatial errors dominate.

5.1. One dimensional problems. In this subsection we test our schemes on one dimensional scalar linear Hamilton-Jacobi equations. The computational domain is $-1<x \leq 1$ and periodic boundary conditions are used for all problems in this subsection.

EXAMPLE 5.1. We test the accuracy of the Hamiltonian corrected fifth order WENO scheme for the linear Hamilton-Jacobi equation

$$
u_{t}+u_{x}=0
$$

with the initial condition

$$
u(x, 0)=\sin (\pi x)
$$

up to $t=2$. The results and a comparison with the original fifth order WENO scheme [9] are given in Table 5.1. We can clearly see that fifth order accuracy is achieved and the errors of the fifth order Hamiltonian corrected WENO scheme are comparable with that of the original fifth order WENO scheme.

EXAMPLE 5.2. We solve the linear Hamilton-Jacobi equation (5.1) with the initial condition

$$
u(x, 0)= \begin{cases}0, & x \leq-0.5 \\ 0.5-|x| & |x|<0.5 \\ 0 & x>0.5 .\end{cases}
$$



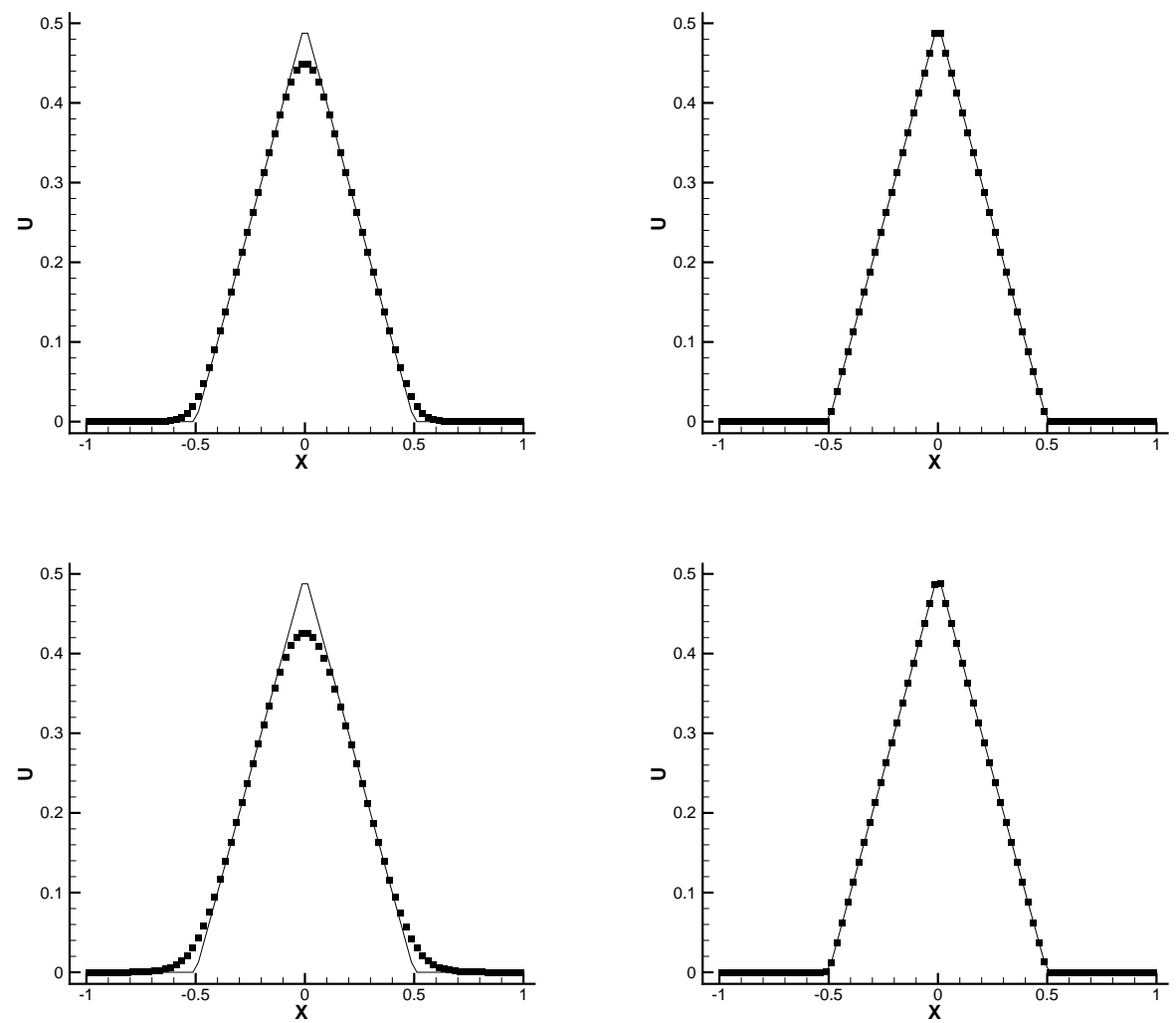

FIG. 5.1. Example 5.2. One dimensional linear Hamilton-Jacobi equation. 80 uniform mesh points. Solid lines: the exact solution; solid rectangle symbols: numerical solution. Left: original fifth order WENO; right: Hamiltonian corrected fifth order WENO. Top: $t=10$, bottom: $t=100$.

The results at $t=10$ (after 5 time periods) and $t=100$ (after 50 time periods) are shown in Figure 5.1. We can see clearly that the regular fifth order WENO scheme progressively smears the kink, while the Hamiltonian corrected fifth order WENO scheme has a sharp resolution for the kink.

ExAmple 5.3. We solve the linear Hamilton-Jacobi equation (5.1) with the initial condition $u(x, 0)=v(x-0.5)$ where

$v(x)=-\left(\frac{\sqrt{3}}{2}+4.5+\frac{2 \pi}{3}\right)(x+1)+ \begin{cases}2 \cos \left(\frac{3 \pi}{2} x^{2}-\sqrt{3}\right), & -1<x \leq-\frac{1}{3} \\ 1.5+3 \cos (2 \pi x), & -\frac{1}{3}<x \leq 0 \\ 7.5-3 \cos (2 \pi x), & 0<x \leq \frac{1}{3} \\ \frac{28+4 \pi+\cos (3 \pi x)}{3}+6 \pi x(x-1) & \text { otherwise. }\end{cases}$

The initial condition of this problem contains kinks and smooth structures. The results at $t=10$ (after 5 time periods) and $t=100$ (after 50 time periods) are shown in Figure 5.2. We can again see better resolution achieved by the Hamiltonian corrected fifth order WENO scheme than that by the regular fifth order WENO scheme. 

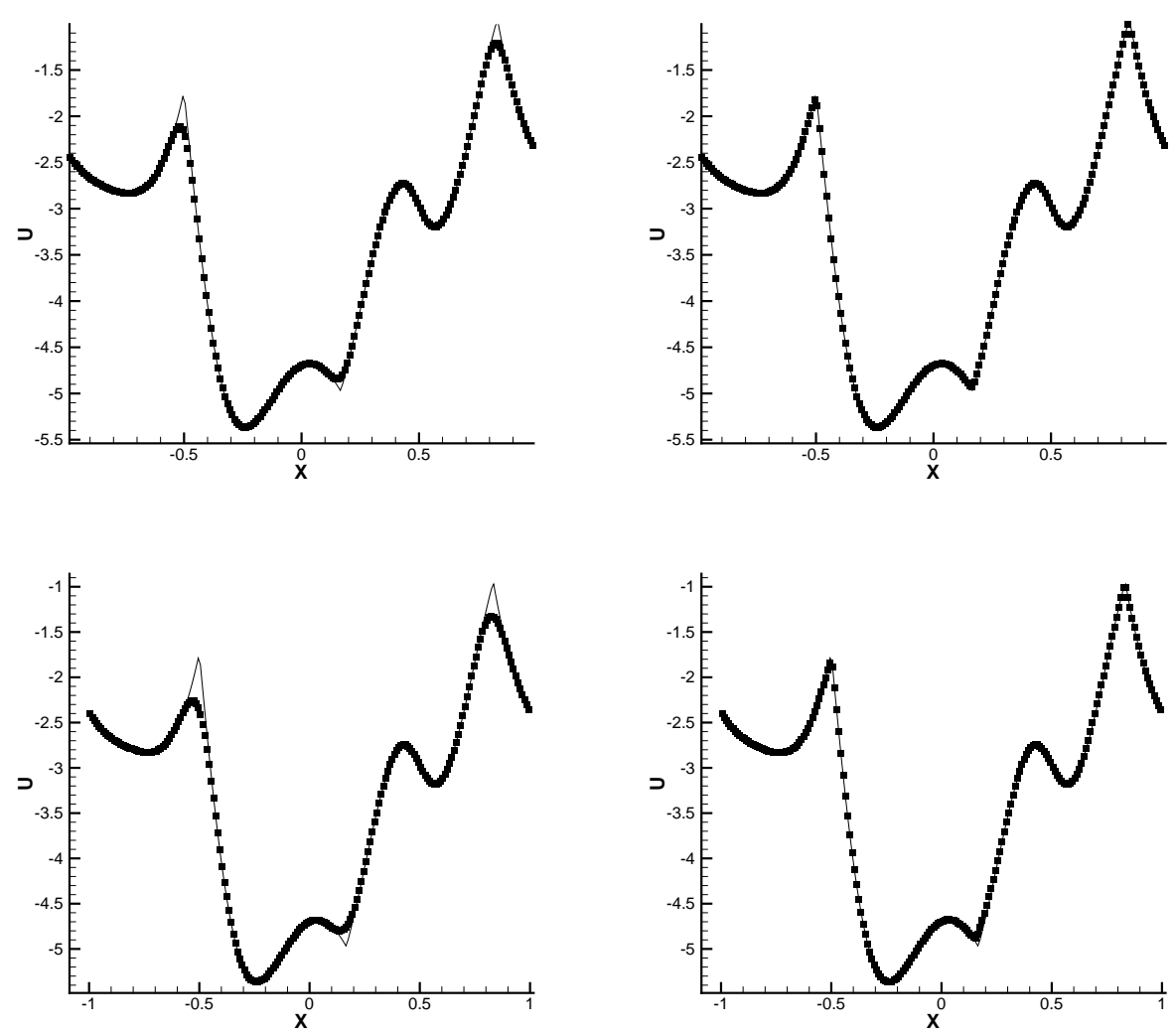

FIG. 5.2. Example 5.3. One dimensional linear Hamilton-Jacobi equation. 200 uniform mesh points. Solid lines: the exact solution; solid rectangle symbols: numerical solution. Left: original fifth order WENO; right: Hamiltonian corrected fifth order WENO. Top: $t=10$, bottom: $t=100$.

5.2. Two dimensional problems. In this subsection we test our schemes on two dimensional linear Hamilton-Jacobi equations. The computational domain is $(x, y) \in[-1,1]^{2}$ with periodic boundary conditions.

EXAmPle 5.4. We solve the two dimensional linear Hamilton-Jacobi equation

$$
u_{t}+u_{x}+u_{y}=0
$$

with the initial condition given by:

$$
u(x, y, 0)= \begin{cases}0, & 0.7 \leq r \\ 0.7-r, & 0.2<r<0.7 \\ 0.5 & r \leq 0.2\end{cases}
$$

with $r=\sqrt{x^{2}+y^{2}}$. The results at $t=2$ (after 1 time period) and at $t=20$ (after 10 time periods) are given in Figures 5.3 and 5.4 for the one dimensional cuts and in Figure 5.5 for the two dimensional surfaces. We can observe a significant improvement of the Hamiltonian corrected fifth order WENO scheme over the regular fifth order 

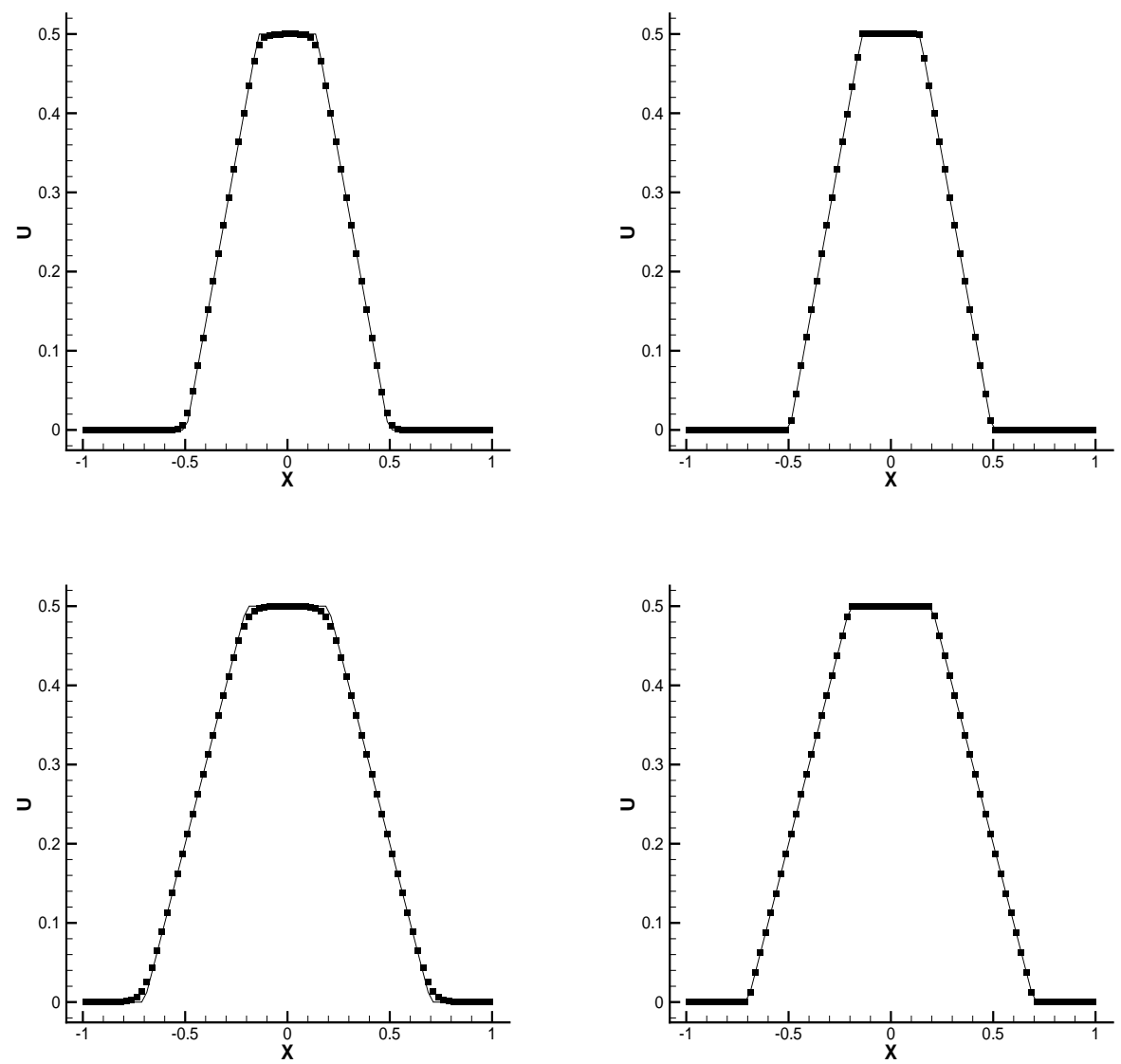

FIG. 5.3. Example 5.4. $80 \times 80$ uniform mesh. $t=2$. Solid line: the exact solution; Filled rectangles: the numerical solution. Top: one dimensional cut of $45^{\circ}$ with the $x$-axis; bottom: one dimensional cut of $0^{\circ}$ with the $y$-axis. Left: regular fifth order WENO scheme; Right: Hamiltonian corrected fifth order WENO scheme.

WENO scheme around the resolution of the kinks, especially for longer time. This improvement can be seen most clearly in the zoomed surface plots in Figure 5.5.

EXAMPLE 5.5. We solve the two dimensional linear Hamilton-Jacobi equation with variable coefficients

$$
u_{t}-y u_{x}+x u_{y}=0
$$

with an initial condition

$$
u(x, y, 0)= \begin{cases}0, & 0.3 \leq r \\ 0.3-r, & 0.1<r<0.3 \\ 0.2 & r \leq 0.1\end{cases}
$$

with $r=\sqrt{(x-0.4)^{2}+(y-0.4)^{2}}$. This is a solid body rotation around the origin. The computational result at $t=2 \pi$ (after one rotation) is given in Figures 5.6 and 

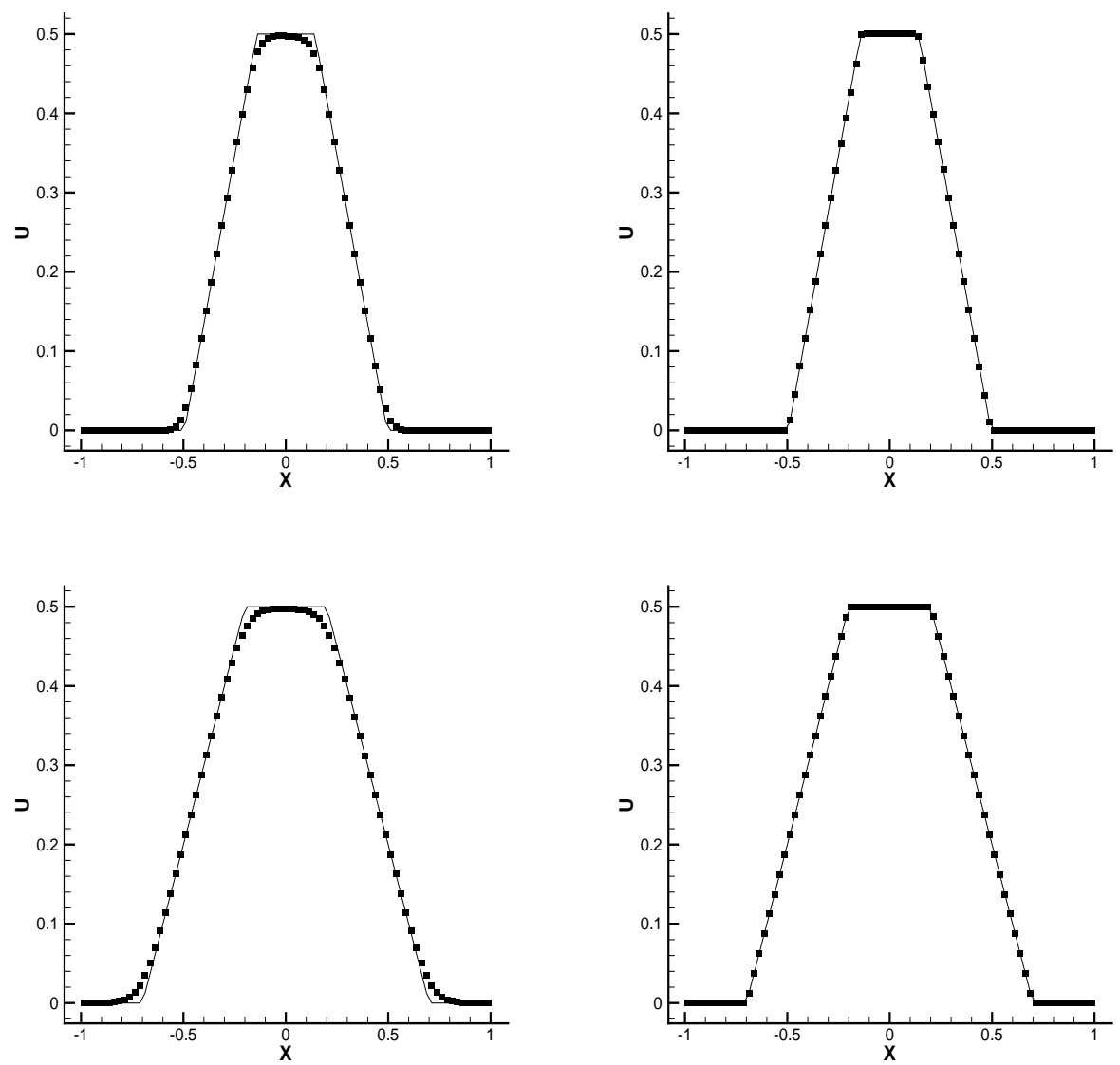

FIG. 5.4. Example 5.4. $80 \times 80$ uniform mesh. $t=20$. Solid line: the exact solution; Filled rectangles: the numerical solution. Top: one dimensional cut of $45^{\circ}$ with the $x$-axis; bottom: one dimensional cut of $0^{\circ}$ with the $y$-axis. Left: regular fifth order WENO scheme; Right: Hamiltonian corrected fifth order WENO scheme.

5.7. We can observe a significant improvement of the Hamiltonian corrected fifth order WENO scheme over the regular fifth order WENO scheme in the resolution of the kinks.

EXAMPLE 5.6. We solve the two dimensional linear Hamilton-Jacobi equation with variable coefficients

$$
u_{t}+f(x, y, t) u_{x}+g(x, y, t) u_{y}=0
$$

with an initial condition

$$
u(x, y, 0)= \begin{cases}0, & 0.3 \leq r \\ 0.3-r, & 0.1<r<0.3 \\ 0.2 & r \leq 0.1\end{cases}
$$

where $r=\sqrt{(x-0.4)^{2}+(y-0.4)^{2}}$. The velocity of the advection depends on $x, y$ and $t$ as $f(x, y, t)=\sin ^{2}(\pi x) \sin (2 \pi y) \cos \left(\frac{t}{T} \pi\right)$ and $g(x, y, t)=$ 
$-\sin ^{2}(\pi y) \sin (2 \pi x) \cos \left(\frac{t}{T} \pi\right)$. The period of deformation is $T=1.5$. This is a numerical test for incompressible flow first introduced by LeVeque in [12]. During the evolution, the initial data is severely deformed, and we can see this in Figure 5.8 at $t=3.75$ (after 2.5 periods). The computational results at $t=1.5$ (after one period) and $t=6.0$ (after four periods) are given in Figures 5.9, 5.10 and 5.11. We can again observe a significant improvement of the Hamiltonian flux corrected fifth order WENO scheme over the regular fifth order WENO scheme in the resolution of the kinks.

6. Concluding remarks. We have generalized the anti-diffusive flux correction techniques developed in [21] for conservation law equations to Hamilton-Jacobi equations. Numerical results both in one space dimension and two space dimensions are given. High order of accuracy of regular WENO schemes for Hamilton-Jacobi equation is maintained, and a sharp resolution of kinks in the solution of linear Hamilton-Jacobi equations is obtained.

\section{REFERENCES}

[1] F. Bouchut, An antidiffusive entropy scheme for monotone scalar conservation law, Journal of Scientific Computing, 21 (2004), pp. 1-30.

[2] B. Cockburn, S. Hou And C.-W. Shu, The Runge-Kutta local projection discontinuous Galerkin finite element method for conservation laws IV: the multidimensional case, Mathematics of Computation, 54 (1990), pp. 545-581.

[3] B. CockBurn AND C.-W. SHU, TVB Runge-Kutta local projection discontinuous Galerkin finite element method for conservation laws II: general framework, Mathematics of Computation, 52 (1989), pp. 411-435.

[4] M. Crandall and P.L. Lions, Viscosity solutions of Hamilton-Jacobi equations, Transactions of American Mathematical Society, 277 (1983), pp. 1-42.

[5] M. Crandall and P.L. Lions, Two approximations of solutions of Hamilton-Jacobi equations, Mathematics of Computation, 43 (1984), pp. 1-19.

[6] B. Després And F. Lagoutière, Contact discontinuity capturing schemes for linear advection and compressible gas dynamics, Journal of Scientific Computing, 16 (2001), pp. 479-524.

[7] A. Harten, B. Engquist, S. Osher and S. Chakravathy, Uniformly high order accurate essentially non-oscillatory schemes, III, Journal of Computational Physics, 71 (1987), pp. 231-303.

[8] C. Hu ANd C.-W. Shu, A discontinuous Galerkin finite element method for Hamilton-Jacobi equations, SIAM Journal on Scientific Computing, 21 (1999), pp. 666-690.

[9] G. Jiang And D. Peng, Weighted ENO schemes for Hamilton-Jacobi equations, SIAM Journal on Scientific Computing, 21 (1999), pp. 2126-2143.

[10] G. JIANG AND C.-W. SHu, Efficient implementation of weighted ENO schemes, Journal of Computational Physics, 126 (1996), pp. 202-228.

[11] O. Lepsky, C. Hu And C.-W. Shu, Analysis of the discontinuous Galerkin method for Hamilton-Jacobi equations, Applied Numerical Mathematics, 33 (2000), pp. 423-434.

[12] R.J. LeVeque, High-resolution conservative algorithms for advection in incompressible flow, SIAM Journal on Numerical Analysis, 33 (1996), pp. 627-665.

[13] F. Li AND C.-W. Shu, Reinterpretation and simplified implementation of a discontinuous Galerkin method for Hamilton-Jacobi equations, Applied Mathematics Letters, 18 (2005), pp. 1204-1209.

[14] X.-D. Liu, S. Osher And T. Chan, Weighted essentially nonoscillatory schemes, Journal of Computational Physics, 115 (1994), pp. 200-212.

[15] S. Osher And J. Sethian, Front propagating with curvature dependent speed: Algorithms based on Hamilton-Jacobi formulations, Journal of Computational Physics, 79 (1988), pp. 12-49.

[16] S. Osher AND C.-W. SHU, High-order essentially nonoscillatory schemes for Hamilton-Jacobi equations, SIAM Journal on Numerical Analysis, 28 (1991), pp. 907-922.

[17] J. ShI, C. Hu AND C.-W. SHu, A technique of treating negative weights in WENO schemes, Journal of Computational Physics, 175 (2002), pp. 108-127.

[18] C.-W. SHU, Essentially non-oscillatory and weighted essentially non-oscillatory schemes for hyperbolic conservation laws, in Advanced Numerical Approximation of Nonlinear Hy- 
perbolic Equations, B. Cockburn, C. Johnson, C.-W. Shu and E. Tadmor (Editor: A. Quarteroni), Lecture Notes in Mathematics, volume 1697, Springer, 1998, pp. 325-432.

[19] C.-W. Shu AND S. Osher, Efficient implementation of essentially non-oscillatory shockcapturing schemes, Journal of Computational Physics, 77 (1988), pp. 439-471.

[20] C.-W. ShU AND S. Osher, Efficient implementation of essentially non-oscillatory shock capturing schemes II, Journal of Computational Physics, 83 (1989), pp. 32-78.

[21] Z. XU AND C.-W. SHU, Anti-diffusive flux corrections for high order finite difference WENO schemes, Journal of Computational Physics, 205 (2005), pp. 458-485.

[22] Y.-T. ZHANG AND C.-W. SHU, High-order WENO schemes for Hamilton-Jacobi equations on triangular meshes, SIAM Journal on Scientific Computing, 24 (2003), pp. 1005-1030. 

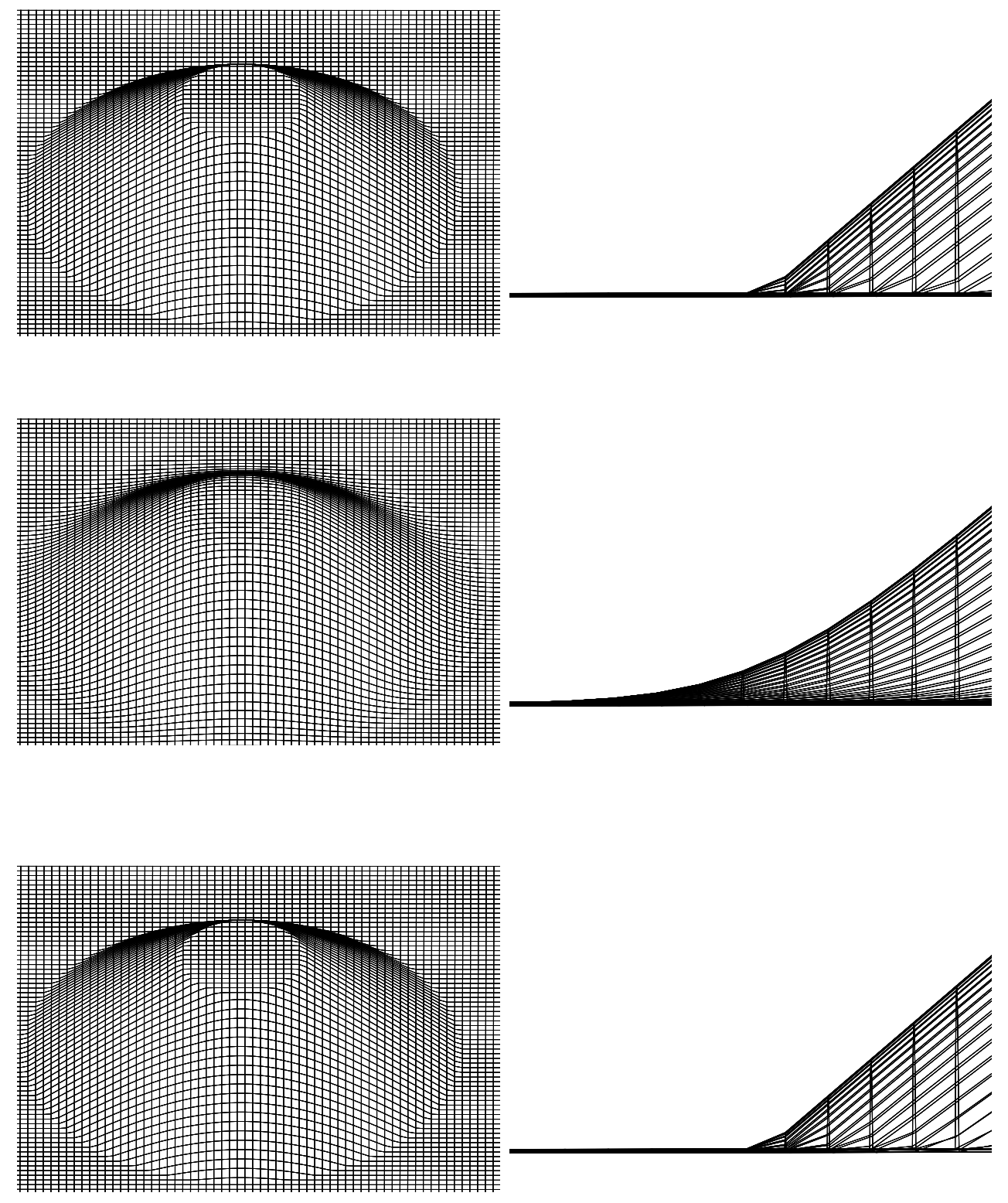

FIG. 5.5. Example 5.4. Surface of $80 \times 80$ uniform mesh. $t=20$. Top: the exact solution; middle: regular fifth order WENO; bottom: Hamiltonian corrected fifth order WENO. Left: full view of the surface; Right: zoomed view of the angle with the $x-y$ plane. 

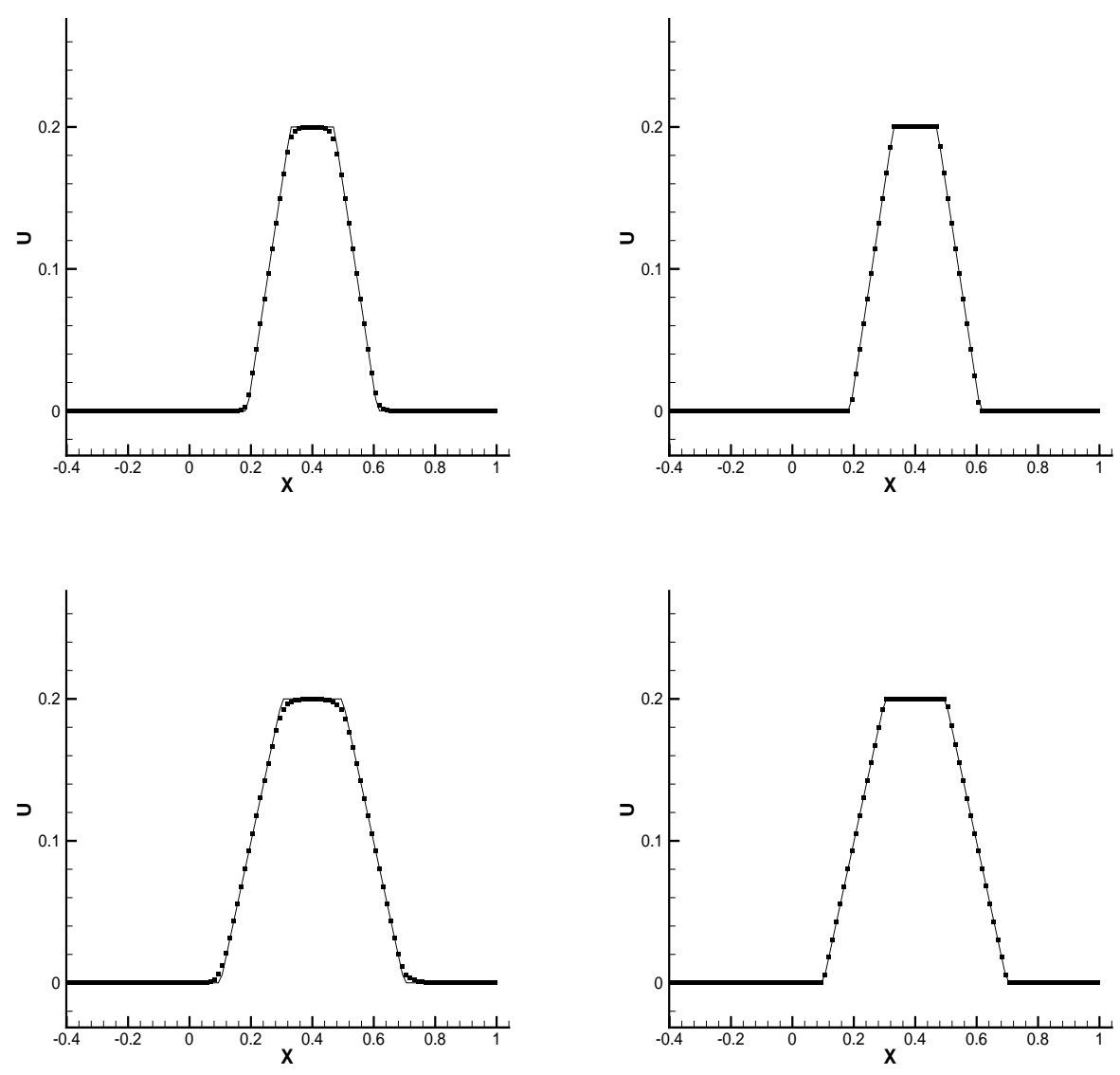

FIG. 5.6. Example 5.5. $160 \times 160$ uniform mesh. $t=2 \pi$. Solid line: the exact solution; Filled rectangles: the numerical solution. Top: one dimensional cut of $45^{\circ}$ with the $x$-axis; bottom: one dimensional cut of $0^{\circ}$ with the $y$-axis. Left: regular fifth order WENO scheme; Right: Hamiltonian corrected fifth order WENO scheme. 

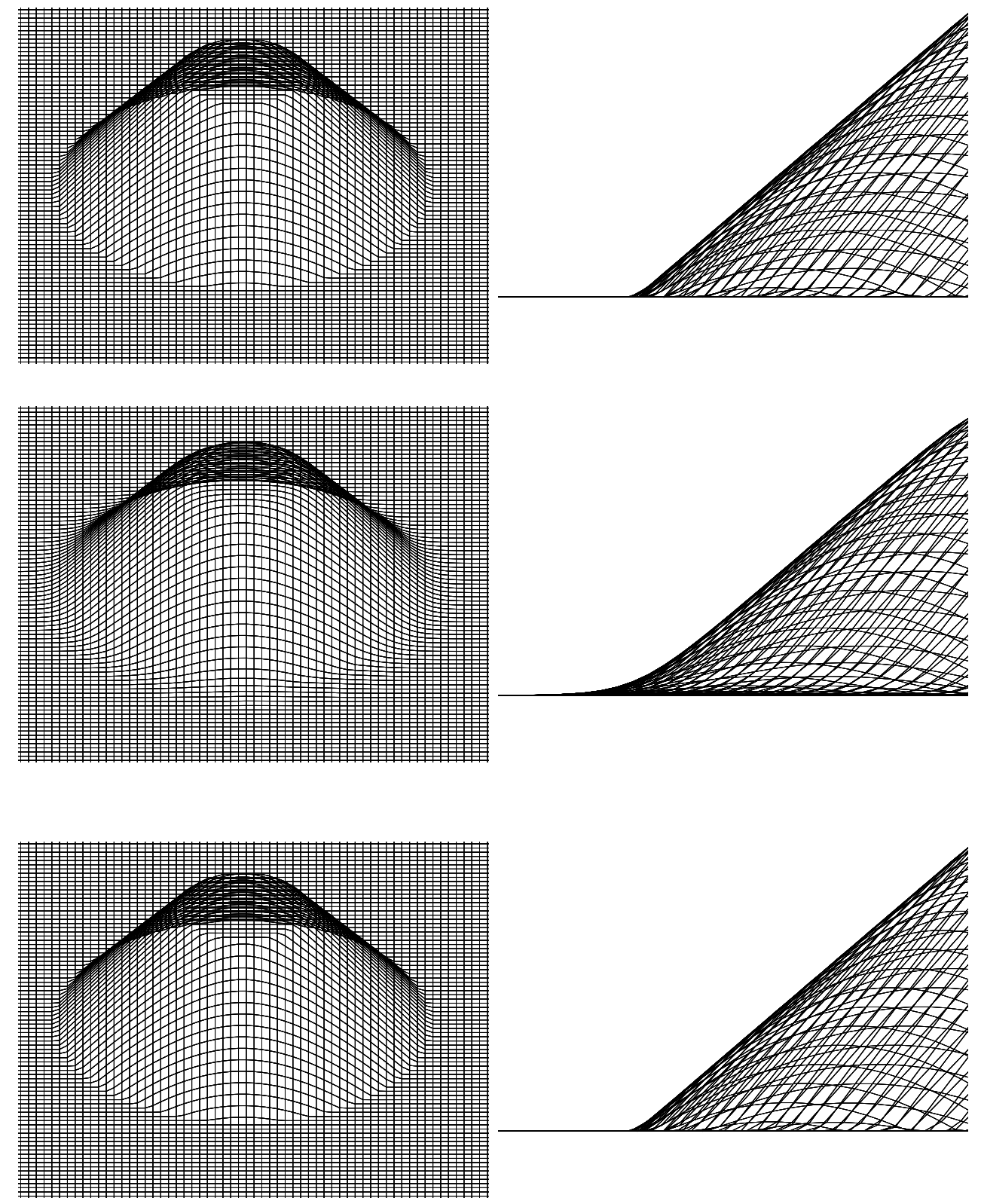

FIG. 5.7. Example 5.5. Surface of $160 \times 160$ uniform mesh. $t=2 \pi$. Top: the exact solution; middle: regular fifth order WENO; bottom: Hamiltonian corrected fifth order WENO. Left: full view of the surface; Right: zoomed view of the angle with the $x-y$ plane. 

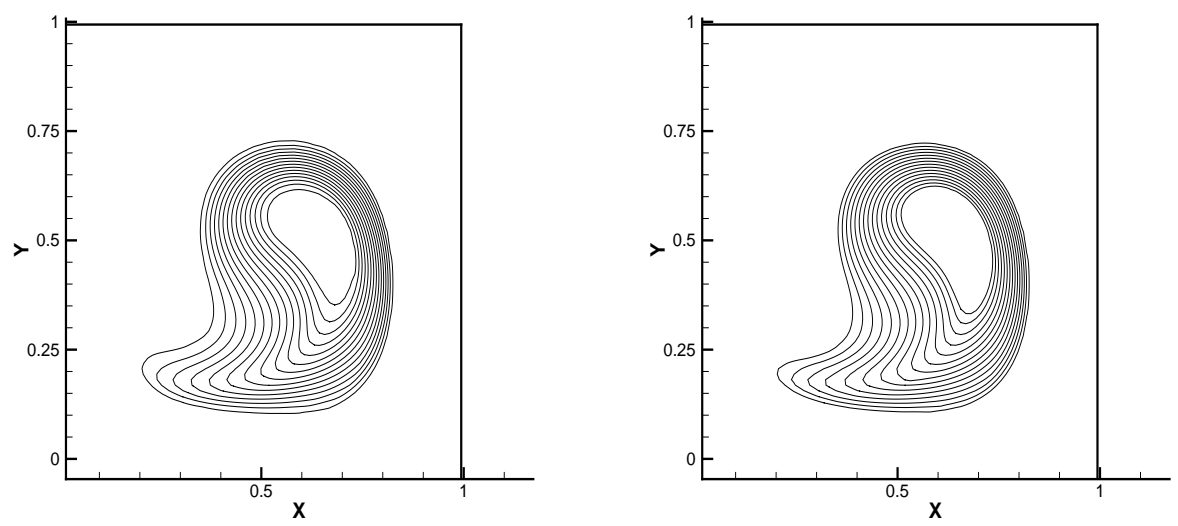

FIG. 5.8. Example 5.6. Contours at $t=3.75$. Left: the regular fifth order WENO scheme; Right: Hamiltonian corrected fifth order WENO scheme. 

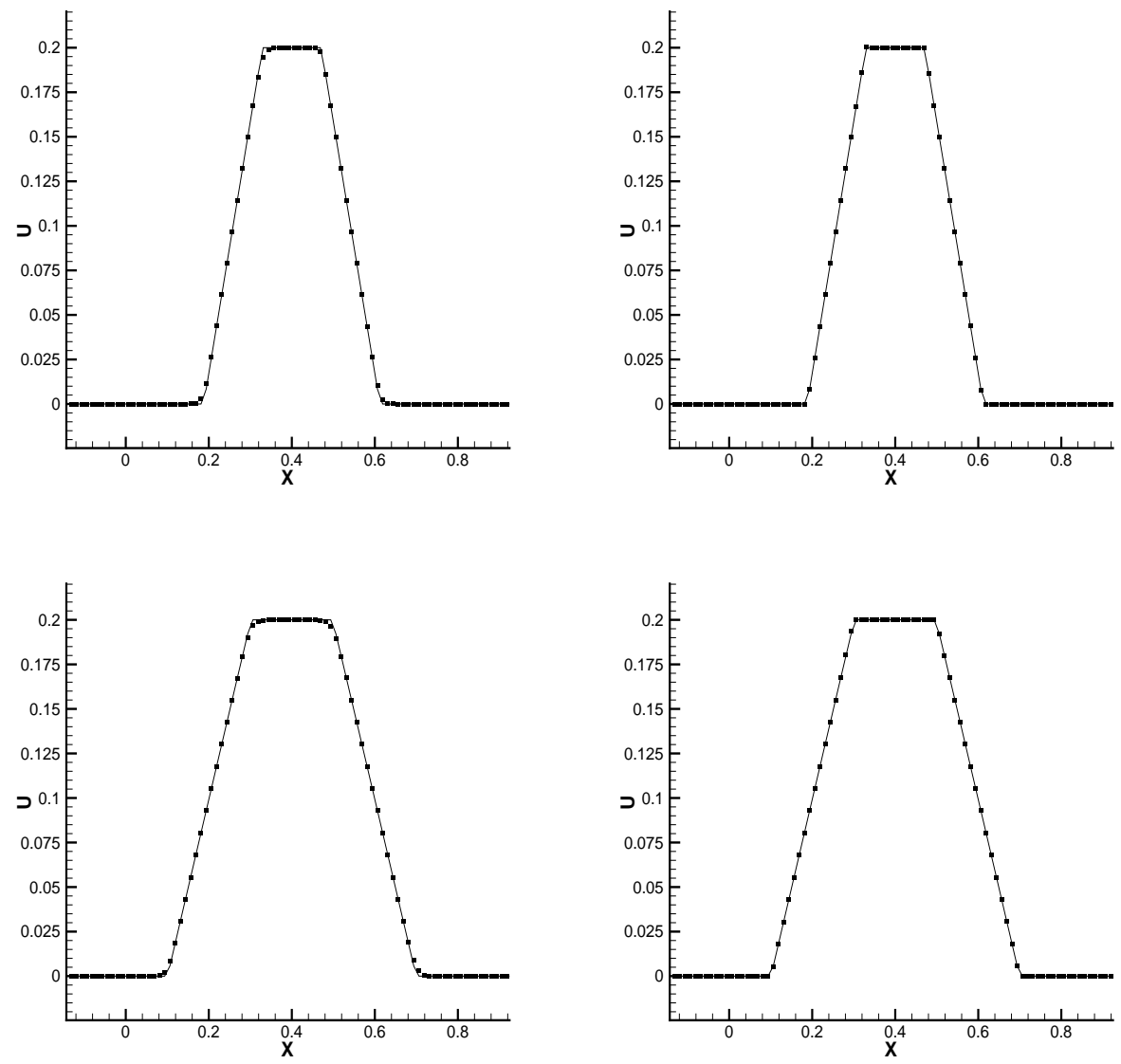

FIG. 5.9. Example 5.6. $160 \times 160$ uniform mesh. $t=1.5$. Solid line: the exact solution; Filled rectangles: the numerical solution. Top: one dimensional cut of $45^{\circ}$ with the $x$-axis; bottom: one dimensional cut of $0^{\circ}$ with the $y$-axis. Left: regular fifth order WENO scheme; Right: Hamiltonian corrected fifth order WENO scheme. 

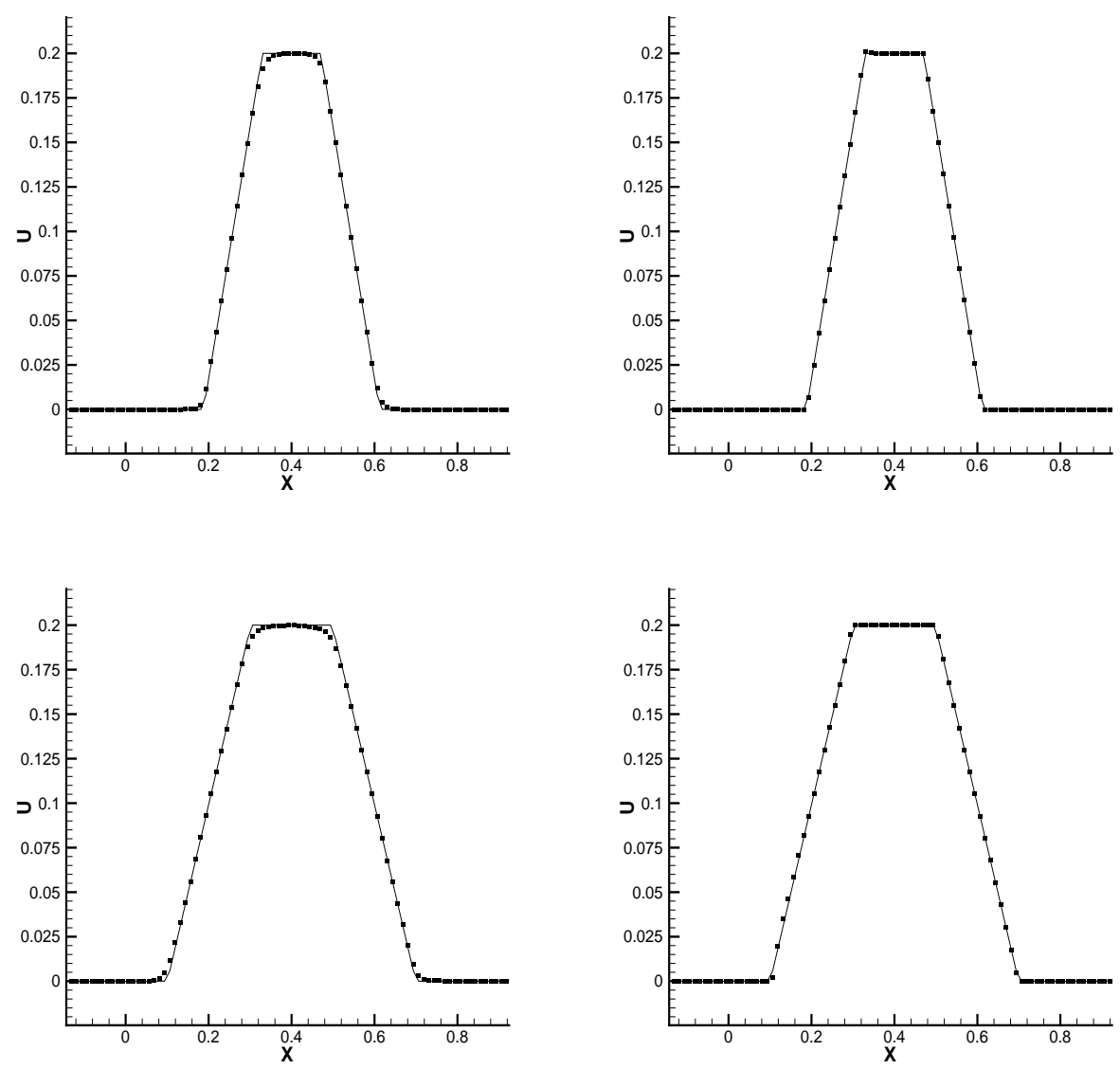

FIG. 5.10. Example 5.6. $160 \times 160$ uniform mesh. $t=6.0$. Solid line: the exact solution; Filled rectangles: the numerical solution. Top: one dimensional cut of $45^{\circ}$ with the $x$-axis; bottom: one dimensional cut of $0^{\circ}$ with the $y$-axis. Left: regular fifth order WENO scheme; Right: Hamiltonian corrected fifth order WENO scheme. 

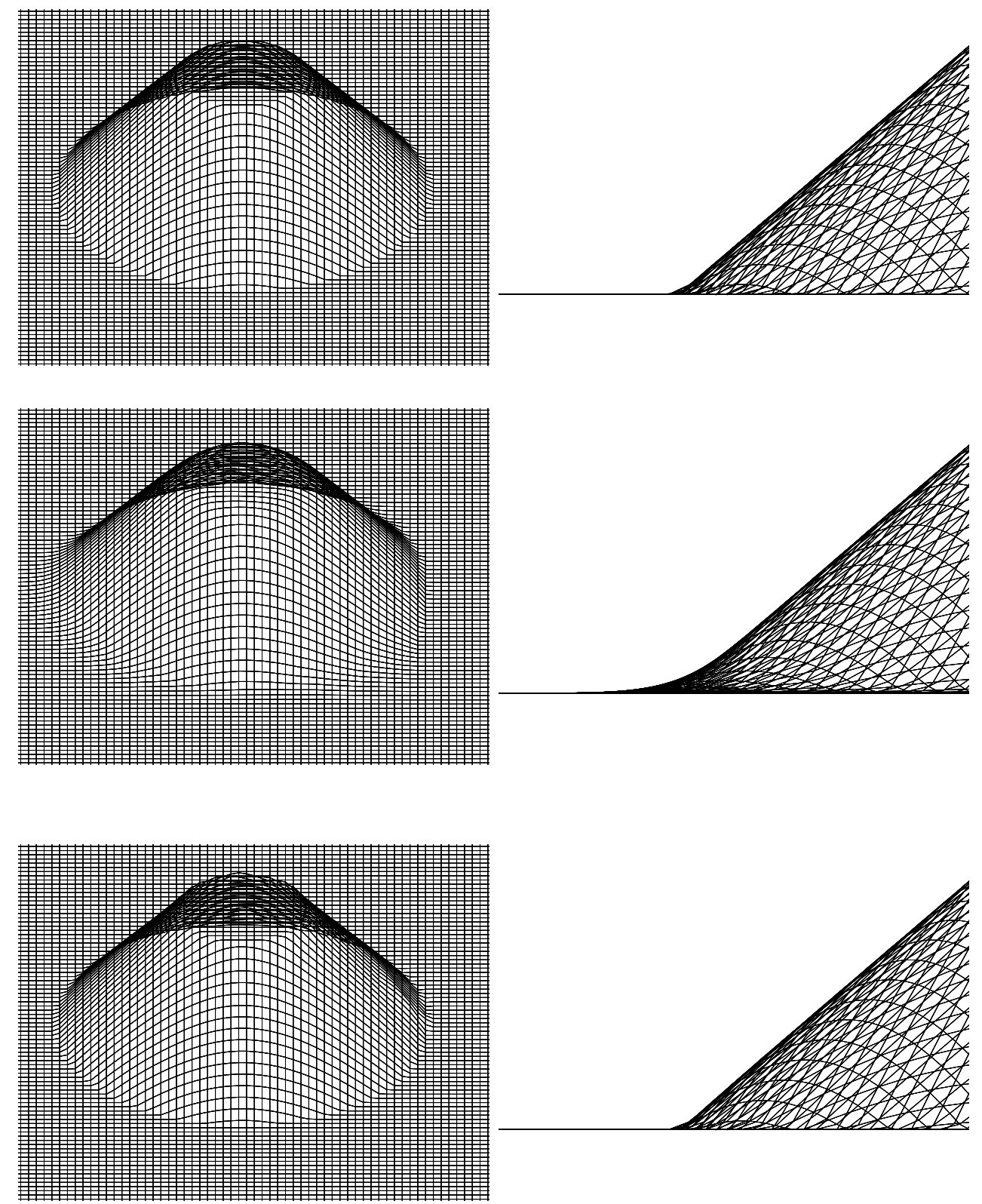

FIG. 5.11. Example 5.6. Surface of $160 \times 160$ uniform mesh. $t=6.0$. Top: the exact solution; middle: regular fifth order WENO; bottom: Hamiltonian corrected fifth order WENO. Left: full view of the surface; Right: zoomed view of the angle with the $x$-y plane. 